\title{
End to End Delay Performance and Comparative analysis of Various TCP Variants in Mobile Ad hoc Networks (MANETs)
}

\author{
Harsimran Kour \\ M.Tech Student \\ Deptt. of ECE \\ MMEC, Mullana, Ambala \\ India
}

\author{
H.P.Sinha, Ph.D \\ Professor \\ Deptt. of ECE \\ MMEC, Mullana, Ambala \\ India
}

\author{
Er. Abhay Bindal \\ Assistant Professor \\ Deptt. of ECE \\ MMEC, Mullana, Ambala \\ India
}

\begin{abstract}
The present age is the age of science and it is not wrong to that almost everybody depend upon science for almost everything. The wide use of wireless network and the various hand held devices increase their importance which results in the popularity of ad hoc network. MANET consists of various mobile nodes that communicate with each other. The reason for congestion in a mobile ad hoc network is not only congestion loss can take place due to various reasons which are BER, multipath routing, other attacks and so on. In this paper the study of various TCP variants has been done which are compared and analyzed on the basis of end to end delay parameter.. The routing protocols which have been taken are AODV, DSR and DSDV and the variants are TCP, TCP SACK, TCP New Reno, TCP Vegas and TCP Westwood. This comparison is done with the help of simulation in NS-2 and the analysis is done on the basis of end to end delay parameter which shows which variant perform better in respective routing protocols and less delay the variant takes the number of packets reaches its destination which means more data reaches the destination. This analysis also helps in better speed, reliability and congestion control
\end{abstract}

\section{Keywords}

AODV, DSR, DSDV, TCP, TCP Variants, MANET.

\section{INTRODUCTION}

Computer networks which are not linked with the help of wires are known as wireless networks and now a day's their use and importance increases because the initial cost of the network reduces significantly [1]. These networks are broadly classified into two categories infrastructure and infrastructure less networks. Infrastructure networks have a central controlling device which is responsible for performing all the functions. Where as in the infrastructure less networks, there present no central controlling device and the nodes itself are responsible for performing the function [2]. Mobile ad hoc network comes under the category of infrastructure less network in which there present no pre existing networks. This network allows to create a network on demand without having a prior configuration and the nodes involve in transmission of the information. The neighboring nodes communicate with each other directly, where as for the non neighboring nodes we require the routing protocol, which helps to send the data between these intermediate nodes. From the two categories of routing protocols, proactive and reactive are much suitable for mobile ad hoc networks due to its ability to cope up with rapidly changing network topologies [3]. There present various challenges and these challenges include routing packets in the environment where the topology is changing frequently and the main task on locating anode and maintain a path to it increases rapidly.TCP (transmission control protocol ) is one of the most important techniques used in the computer networks foe the efficient and reliable transmission of data over the network. It generally complements internet protocol. This protocol is generally used and implemented by many internet applications such as file transfer, World Wide Web, electronic mailing, etc. The transmission of data is very difficult task by using TCP. Congestion Control is an approved mechanism that detects an optimal bandwidth for transmitting data segments over networks.tcp is a connection oriented protocol. Transport Control Protocol /Internet Protocol (TCP/IP) is a connection oriented protocol of the transport layer [4]. Now TCP is most extensively used protocol as it tuned to provide high-quality performance in the conventional wired network. It provides features like flow control, reliability and congestion control. It has been very effective in data delivery and transmission have also developed variants to possess the possibility to increase performance and multiple packet loss recovery [6].

However in case of MANET, it cannot offer reliable and efficient service while using e-mail, internet search and file transmission. This protocol is a standard networking protocol on the internet and is the most widely used transport protocol for data services like file transfer, e-mail and www browser. It is designed for wire line networks, but when used in ad hoc networks, there takes place various degradation problem, but the traditional TCP believes that the reason for degradation is congestion where as in case of MANET the reason for data loss or degradation is not congestion there present various more reasons which leads to this problem and these problems are BER, multi path routing, packet drop and so on. So we use differ TCP variants in which different routing protocols behave differently. It is important to understand the different MANET routing protocols under TCP variants [6].

\section{ROUTING PROTOCOLS}

Routing is defined as the path which helps in the transmission of data between the intermediate nodes. Routing protocols are broadly classified into three categories, reactive proactive and hybrid routing protocol. This paper consists of the two categories i.e reactive and proactive routing protocols which include AODV,DSR and DSR from which AODV,DSR comes under reactive routing protocol and DSDV comes under proactive routing protocol. 


\subsection{AODV}

It stands for ad hoc on demand vector routing protocol. Each Node maintains only the next hop information on the route to the destination. Destination sequence number is used to check the freshness of the route to destination. Periodic use of Beacons i.e. Hello packets used to check the presence of the neighbor. Each node uses a sequence number which is increased whenever the node observe a change in neighbor topology. Each node maintains a routing table and the information is stored as

$<$ Destination IP address, Destination Sequence number, Next hop address, hop count to destination> [10].

\subsection{DSR}

It stands for dynamic source routing. The basic idea of DSR is that, it uses the concept of source routing where the sender knows the complete hop-by hop route to the destination. In this protocol, all the mobile nodes are required to maintain route caches which contain the routes to other nodes. When any new route The route cache is updated when a new route is entered into the cache The data packets carry the source route in the packet header. DSR depends upon two procedures which are

\section{- Route discovery}

- Route maintenance

Suppose there are a source node and wants to send a data packet to a destination, it firstly checks its cache whether its route is present or not and if it contains the route to destination then it uses the same route for the data transmission. If not, then the source node broadcasts a route request packet which includes the destination address, source address, and a unique request ID. Each intermediate node checks whether the route is available or not. If the non neighboring node does not know the route to destination, it gives its own address to route request packet and forwards the packet and to other nodes eventually it reaches the destination. The node processes the route request packet only if it is not previously processed that packet. A route reply is generated by the destination or by any of the intermediate nodes which knows the route to destination.

Another Phase is Route Maintenance which is done by using the route error packet (RERR) and acknowledgements. Route error packets are generated by a node if there is any Link break occurs or any other error in the route. When a route error packet is received by the node, the hop in error is removed from the route cache [10].

\subsection{DSDV}

It stands for destination sequence distance vector routing protocol. It is a table driven routing protocol. Each of the routing table includes all available destinations and the number of hops to reach that destination. Each entry in the routing table has a sequence number. If a Link is present, then sequence number will be even otherwise the odd number will be used. DSDV protocol each mobile node in the network will send its routing table to its current neighbors. This is possible by two processes either by broadcasting or by multicasting. With the help of advertisements, the neighboring nodes can know whether any change has occurred in the network due to the movements of nodes. The routing updates can be sent in two ways, one is called a "full dump" and another is ' 'incremental.' In full dump, the entire routing table is transmitted to the neighbors, when change occurs in the topology. But in case of incremental update only the entries that are updated due to changes are sent [10].

\section{TCP VARIANTS}

\subsection{TCP New Reno}

TCP-New Reno is modified form of Reno with an improved Fast Recovery (FR) algorithm in order to solve the time out problem where multiple packets are lost from the same window. It is capable of detecting the multiple packet loss in the network .Congestion Control components of TCP-New Reno and TCP-Reno are identical. TCP- New Reno distinguishes a Full ACK (FA) from a Partial ACK (PA)by modifying TCP-Reno's Fast Recovery behavior after it receives a non-duplicate ACK. All the outstanding segments are fully acknowledged at the beginning of FR by FA where as PA acknowledges only some of the outstanding data. TCP New Reno unlike Reno can recover from multiple segment losses by retransmitting only one lost segment in the same window per RTT and remains in Fast Recovery unless and until a full ACK is received [13].

\subsection{TCP Sack}

Selective Acknowledgement (SACK) also encounters the problem of multiple packet losses like new Reno. Here the acknowledgement is only provided for the selected segments which have been received successfully. TCP-SACK thus requires retransmission of only those segments that has not yet been acknowledged. So it reduces the number of retransmissions required by the network. Each acknowledgement contains information of up to three non contiguous blocks received by the sender. The same fast recovery procedure is used by both TCP SACK and TCP which activate Congestion Avoidance algorithm even for a single packet loss. For situations where multiple packet losses occur in an outstanding data window, TCP-Sack outperforms standard TCP. This scheme of SACK is not efficient for small sized window [13].

\subsection{TCP Vegas}

The scheme used by Vegas to estimate the bandwidth is more efficient as compared to the other TCP variants. This scheme makes bandwidth estimation by using the difference between the expected flow rates and the actual flow rates. It extends TCP-Reno by modifying its Congestion Avoidance mechanism. Like TCP-Reno it uses Slow Start and Fast Retransmission. TCP-Vegas use its Congestion Avoidance mechanism in order to avoid packet loss by decreasing its CWND as soon as it detects congestion in the network. This is unlike TCP-Reno which initiates its Congestion Avoidance mechanism and increases its CWND until a packet loss is detected in the network. Also Retransmission mechanism used by TCP-Vegas is more efficient as compared to TCP-Reno as it retransmits the corresponding packet as soon as it receives a single duplicate ACK and does not wait for three ACKs. TCPVegas as compared to TCP-Reno is more accurate and is less aggressive, thus it does not reduce its CWND unnecessarily [13].

\subsection{TCP Westwood}

TCP-Westwood (TCPW) simply modifies the TCP source protocol stack, which helps the source to estimate the available bandwidth of the connections. It estimates the bandwidth by calculating the rate at which data is being delivered to the destination. The delivery rate is calculated from the ACK 
information and the rate with which the ACKs are being received. It then uses the estimated bandwidth for fast recovery of the lost packets which results in improved throughput. Unlike TCP-Reno which halves CWND size aggressively after a packet loss, TCP $\mathrm{W}$ tries to select an appropriate ssthresh and CWND size which provides an effective connection rate during the congestion. Thus, it handles the packet losses effectively by utilizing the information of current CWND size and ssthresh [13].

\section{IMPLEMENTATION}

The tool used for the implementation is Ns2 (network simulator) Ns-2 means Network simulator version 2. It is a discrete event simulator where the activities got converted into events which are then processed with respect to their respective timing at the time progresses the events processed and this simulation time is not the real life time. This simulator provides us the whole view of network construction. Experimental set up consists of 50 mobile nodes which are places in a mobile ad hoc network having an area of $1000 \times 1000$ meters having the simulation time of 25 seconds. It consists of an omnidirectional antenna which radiates in all the directions and also here present MAC /802.11. Three routing algorithms have been used which are AODV, DSR and DSDV and the interface queue length is 50 and it is used for each node. Here node 0 is the source node and node 49 is the destination node. Data transmission takes place between these nodes following the different routing paths.

\section{RESULTS AND DISCUSSION}

The comparison of different TCP variants can be done on the basis of end to end delay parameter and the analysis of these variants can be done with the help of $\mathrm{x}$ graph which shows end to end delay of five different TCP variants which include TCP, TCP New Reno, TCP SACK, TCP Vegas and TCP Westwood and their performance is analyzed in three different routing protocols which shows us which variant perform better in respective routing protocols.

\subsection{End to end delay}

It is defined as the time taken for a packet to be transmitted across the network from source to destination.

Table1.Simulation parameters

\begin{tabular}{|c|c|}
\hline Method & Value \\
\hline Channel type & Channel/wireless channel \\
\hline Propagation model & Propagation/TwoRayGround \\
\hline Netwrk interface & Phy/WirelessPhy \\
\hline MAC type & MAC/802.11 \\
\hline Interface queue type & Queue/Drop tail/PriQueue \\
\hline Link layer type & LL \\
\hline Antenna & Antenna/Omni antenna \\
\hline Max. Packets in ifq & 50 \\
\hline Area(m×m) & $1000 \times 1000$ \\
\hline Number of nodes & 50 \\
\hline Simulation time & 25 sec \\
\hline Routing protocol & AODV, DSR,DSDV \\
\hline Parameter & End to end delay \\
\hline
\end{tabular}

The above figure shows the various simulation parameters which are used for the simulation. The metric on which the variants are compared is end to end delay and the various simulation results are as shown below.

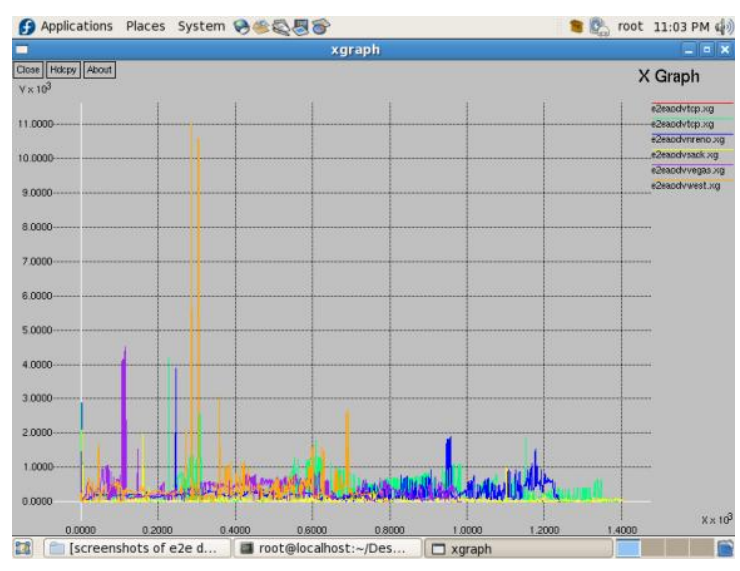

Fig 1: Comparison of Different TCP Variants on the Basis of End to End Delay in Case of AODV Routing Protocol.

In the figure 1 the end to end delay of different variants have been shown in AODV routing protocol and from the graphical representation it has been seen that TCP sack has less delay as compared to other variants. The graph is between the number of packets and the time so during the very first packet TCP sack takes $2 \mathrm{sec}$ and its time changes when the number of packet increases, but after 1100 packet was transmitted its delay become constant and for remaining packets they present no delay where as from 400 to 1200 packets the end to end to delay seems to be very high for TCP variants except TCP Sack. So from all the five variants TCP Sack shows better end to end delay in AODV.

Table2.Showing the Comparison Of Different TCP Variants on the Basis of End to End Delay in Case of AODV Routing Protocol.

\begin{tabular}{|l|l|l|l|}
\hline $\begin{array}{l}\text { Name of } \\
\text { Protocol }\end{array}$ & $\begin{array}{l}\text { TCP } \\
\text { variants }\end{array}$ & $\begin{array}{l}\text { Packet } \\
\text { number } \\
\mathbf{\times 1 0 ^ { \wedge } 3}\end{array}$ & $\begin{array}{l}\text { Delivery } \\
\text { time } \\
(\mathbf{s e c}) \\
\times \mathbf{1 0}^{\wedge} \mathbf{3}\end{array}$ \\
\hline AODV & TCP Sack & 1 & 47.959 \\
\hline AODV & $\begin{array}{l}\text { TCP } \\
\text { Westwood }\end{array}$ & 1 & 206.771 \\
\hline AODV & TCP & 1 & 267.129 \\
\hline AODV & $\begin{array}{l}\text { TCP } \\
\text { Vegas }\end{array}$ & 1 & 267.726 \\
\hline AODV & $\begin{array}{l}\text { TCP New } \\
\text { Reno }\end{array}$ & 1 & 288.981 \\
\hline
\end{tabular}

In the table 2 it has been seen that as the end to end delay is the time taken by the packet to reach its destination. Here when the data transmission takes place then comparing the transmission of packet number 1 in case of different TCP variants in same AODV routing protocol it has been observed that the least time taken by the packet to reach its destination 
is $47.959 \times 10^{\wedge} 3 \mathrm{sec}$ and this taken is taken by TCP sack in case of AODV rotting protocol which means that TCP sack has effective data transmission capability as compared to other TCP variants and also the packets in case of TCP sack data reaches much faster to its destination as compared to other variants as it take less time and thus more data reaches its destination. Thus, TCP SACK has better end to end delay in case of AODV routing protocol as compared to other TCP variants.

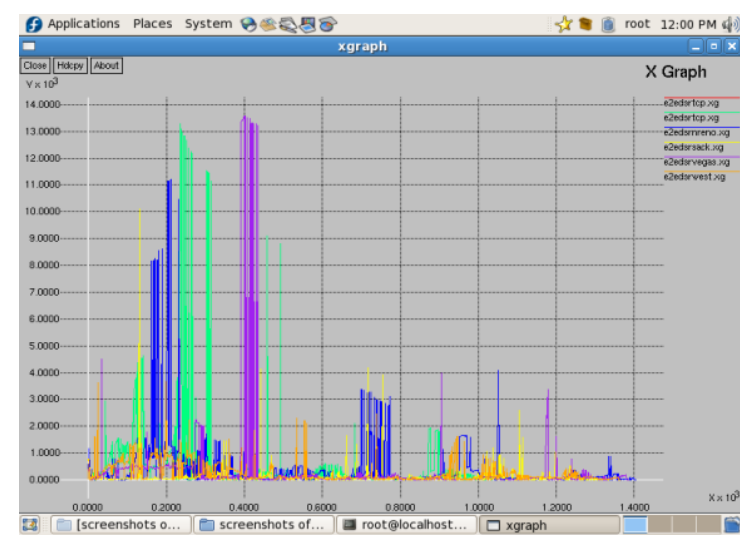

Fig 2:- Comparison of Different TCP Variants on the Basis of End to End Delay in Case of DSR Routing Protocol.

Figure 2 shows the comparison of TCP variants on the basis of end to end delay in the DSR routing protocol. Figure shows that end to end delay seems to be in the very high rate when the packet number from 100 to 800 has been transmitted, but in case of TCP West wood the end to end delay is very less as compared to other TCP variants and after $800^{\text {th }}$ packet has been transmitted the TCP west wood's end to end delay remains stable and shows very less end to end delay which means that the in case of DSR routing protocol TCP West wood has better end to end delay as compared to other TCP variants.

Table3.Showing the Comparison Of Different TCP Variants on the Basis of End to End Delay in Case of DSR Routing Protocol.

\begin{tabular}{|l|l|l|l|}
\hline $\begin{array}{l}\text { Name of } \\
\text { Protocol }\end{array}$ & $\begin{array}{l}\text { TCP } \\
\text { variants }\end{array}$ & $\begin{array}{l}\text { Packet } \\
\text { number } \\
\times \mathbf{1 0}^{\wedge} \mathbf{3}\end{array}$ & $\begin{array}{l}\text { Delivery time } \\
(\mathbf{s e c}) \times \mathbf{1 0} \mathbf{3}\end{array}$ \\
\hline DSR & $\begin{array}{l}\text { TCP } \\
\text { Westwood }\end{array}$ & 1 & 600.783 \\
\hline DSR & TCP Vegas & 1 & 611.336 \\
\hline DSR & TCP & 1 & 777.475 \\
\hline DSR & $\begin{array}{l}\text { TCP New } \\
\text { Reno }\end{array}$ & 1 & 849 \\
\hline DSR & TCP Sack & 1 & 1157.72 \\
\hline
\end{tabular}

From the table 3 it has been seen different TCP variants have been compared on the basis of end to end delay in case of DSR routing protocol. As the transmission rate of packet number 1 is compared and it has been observed that in case of TCP Westwood their present less error that's why the data reaches it destination much faster as compared to the other TCP variants. The table shows the ascending order of TCP variants in case of end to end delay less time the variant takes for the data transmission more data reaches the destination which means that less error takes place which meant that less data loss takes place.

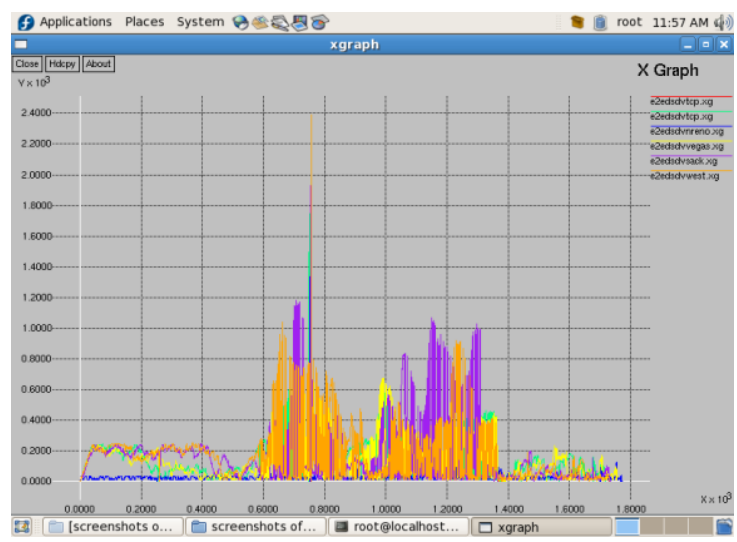

Fig 3: Comparison of Different TCP Variants on the Basis of End to End Delay in Case of DSDV Routing Protocol.

Figure shows the end to end delay comparison analysis of different TCP variants in DSDV routing protocol and from the figure it seems that in case of DSDV TCP shows better end to end delay as compared to other variants as from the figure it was seen that form the packet number 600 to 1400 the there present large delay and these packets reach the destination having some delay but in case of TCP New Reno its delay time is very less as compared to other variants and form the figure 3 it has been seen that the packets take the constant time to reach the destination which means that there present less delay in TCP New Reno as compared to other variants in case of DSDV routing protocol.

Table4.Showing the Comparison Of Different TCP Variants on the Basis of End to End Delay in Case of DSDV Routing Protocol.

\begin{tabular}{|l|l|l|l|}
\hline $\begin{array}{l}\text { Name of } \\
\text { Protocol }\end{array}$ & $\begin{array}{l}\text { TCP } \\
\text { variants }\end{array}$ & $\begin{array}{l}\text { Packet } \\
\text { number } \\
\mathbf{\times 1 0 ^ { \wedge } \mathbf { 3 }}\end{array}$ & $\begin{array}{l}\text { Delivery } \\
\text { time } \\
(\mathbf{s e c}) \mathbf{\times 1 0} \mathbf{3} 3\end{array}$ \\
\hline DSDV & $\begin{array}{l}\text { TCP New } \\
\text { Reno }\end{array}$ & 1 & 4.83603 \\
\hline DSDV & TCP & 1 & 5.09603 \\
\hline DSDV & $\begin{array}{l}\text { TCP } \\
\text { Westwood }\end{array}$ & 1 & 5.17603 \\
\hline DSDV & TCP Sack & 1 & 5.91603 \\
\hline DSDV & $\begin{array}{l}\text { TCP } \\
\text { Vegas }\end{array}$ & 1 & 12.816 \\
\hline
\end{tabular}

From the table 4 it has been observed that different TCP variants have been compared on the basis of end to end delay parameter in case of DSDV routing protocol. Here the 
comparison can be done on the basis of time taken by the packet to reach its destination. Here the comparison is done for the packet number 1 and it has been seen that TCP New Reno takes very less time for the packet to reach its destination which means that more data reaches the destination in less time as compared to other TCP variants. For the packet number 1 it has been observed that the time taken by the packet is 4.83603 which is less as compared to other TCP variants so in case of DSDV routing protocol, TCP New Reno has less end to end delay which means that it take less time for the packet to reach its destination which resukts in more packets to reach its destination.

\section{CONCLUSION}

The proposed work shows the simulation of five tcp varints :TCP,TCP New Reno, Sack, Vegas and westwood over the three routing protocols which are AODV,DSR,DSDV in MANET. Simulation shows 50 nodes and the transmission of data takes place between these nodes.here the variants are compared on the basis of end to end delay and the analysis is done by using xgraph. The implementation is performed in NS2. From the above results it has been show that in case of AODV routing protocol TCP Sack shows better end to end delay as compare to the other tcp variants, which means that packets reach the destination in much less time in case if SACK as compare to other TCP and due to which more data will reach the destination. In case of DSR, TCP west wood shows less delay and in case of DSDV TCP New Reno shoes less delay as compared to other TCP variants. From the above analysis, it has been found that less delay the packet takes more fast it reaches its destination due to which more data will reach the destination. From the table 2,3 and 4 its has been seen the different time taken by the TCP variants for the transmission of packet number 1 in case of different routing protocols. The tabular form easily shows the comparison of different TCP variants in case of end to end delay and from these tables it has been seen that in case of AODV routinbg protocoil TCP Sack shows better end to end delay means it takes very less for the packet to reach its destination and time taken by TCP Sack is $47.959 \times 10^{\wedge} 3$ where as in cxase of DSR routing protocol time taken by TCP Westwood is $600.783 \times 10^{\wedge} 3$ and in case of DSDV routing protocol time taken by TCP New Reno is $4.83603 \times 10^{\wedge} 3$ so it shows that for the same parameter different TCP variants show better performance in case of different routing protocol for better speed, relaibility and better data transmission to avoid comngestion p;roblem.

\section{REFRENCES}

[1] Neeraj Sharma, Manish Mann, Ravinder Thakur, "TCP Congestion Control in Wired cum Wireless Networks", International Journal of Computer Applications (0975 8887) Volume 88- No.5, pp: 34-37, February 2014.

[2] Heena Dave, Vikas Gupta, Parul Dihulia, "Performance Comparison between TCP Sack and TCP Vegas using NS-2 Simulator", International Journal of Computer Applications , ISSN(0975 - 8887), Volume 68- No.11, pp: 49-52 April 2013.

[3] Gayathri Janakiraman, T. Nirmal Raj, R.M. Suresh, "AODV, DSDV, DSR Performance Analysis with TCP Reno, TCP New Reno, TCP Vegas on Mobile Ad-hoc
Networks using NS2", International Journal of Computer Applications (0975 - 8887) Volume 72- No.19, pp:1-7, June 2013.

[4] Hrituparna Paul, Priyanka sarkar, " A Survey: High Speed TCP Variants in Wireless Networks", International Journal of Advance Research in Computer Science and Management Studies, Volume 1, Issue 7, ISSN: 23217782, pp: 142-148, December 2013.

[5] Hrituparna Paul, Anish Kumar Saha, Partha Pratim Deb, Partha Sarathi Bhattacharjee "Comparative Analysis of Different TCP Variants in Mobile Ad-Hoc network", International Journal of Computer Applications (0975 8887) Volume 52- No.13, August 2012.

[6] Harpreet Singh Chawla, M. I. H. Ansari, Ashish Kumar, Prashant Singh Yadav, "A Survey of TCP over Mobile ADHOC Networks", International Journal of Scientific \& Technology Research, Volume 1, Issue 4, ISSN 22778616, pp:146-150, May 2012

[7] Shimaa Hagag , Ayman El-Sayed, "Enhanced TCP Westwood Congestion Avoidance Mechanism (TCP WestwoodNew", International Journal of Computer Applications, ISSN (0975 - 8887), Volume 45- No.5, pp:21-19,May2012.

[8] Suhas Waghmare et. al "Comparative Analysis of different TCP variants in a wireless environment", 978-1-42448679-3/11 @2011 IEEE.

[9] Pratap K. Meher, P. J. Kulkarni, "Analysis and Comparison of Performance of TCP-Vegas in MANET", International Conference on Communication Systems and Network Technologies, IEEE, ISSN 978-0-76954437-3, pp: 67-70, 2011.

[10] Kavita Pandey, Abhishek Swaroop, “A Comprehensive Performance Analysis of Proactive, Reactiveand Hybrid MANETs Routing Protocols", International Journal of Computer Science Issues (IJCSI), Vol. 8, Issue 6, No 3, ISSN (1694-0814), pp: 432-441, November 2011.

[11] Md. Monzur Morshed; Franz I. S. Ko; Dongwook Lim; Md. [10]Habibur Rahman; Md. Rezaur Rahman Mazumder; Jyotirmoy Ghosh, "Performance evaluation of DSDV and AODV routing protocols in mobile ad-hoc networks", NISS, 2010, pp. 399-403, May 2010.

[12] V.Ramesh, Dr.P.Subbaiah, N. Koteswar Rao, M.Janardhana Raju, "Performance Comparison and Analysis of DSDV and AODV for MANET", (IJCSE) International Journal on Computer Science and Engineering, ISSN : 0975-3397, Vol. 02, No. 02, pp: 183-188, 2010.

[13] Shagufta Henna, "A Throughput Analysis of TCP Variants in MobileWireless Networks"Third International Conference on Next Generation Mobile Applications, Services and Technologies, IEEE, ISSN: 978-0-7695-3786-3/09, 2009.

[14] NS Simulator for beginners, http://www/sop.inria.fr/members/Eitan.Altman/COURSNS/n3.pdf. 